\title{
Programmatically Convert Printable Document to Flash File on Web- based Instruction Platform
}

\author{
Zhaohui Lu ${ }^{\mathrm{a}}$, Liming Xie ${ }^{\mathrm{b}}$, Wenjuan Jiang ${ }^{\mathrm{c}}$ \\ ${ }^{a, b, c}$ Network Center, Hainan Normal University, Haikou, China
}

\begin{abstract}
Seeing the existing problems of currently known web-based instruction platform and the benefits of flash file, this paper brings up a solution which combines website module with desktop application module based on command-line utility flashprinter.exe and third-party application software, so as to programmatically convert printable documents to flash files, and then detailedly presents the partial main implementation and some runtime interfaces. In the end, the paper draws a conclusion that this solution is applied to web-based instruction platform.
\end{abstract}

Index Terms: Converrt; Printable Document; Flash File; Flashpaper; Web Fom; Win Form

(C) 2012 Published by MECS Publisher. Selection and/or peer review under responsibility of the International Conference on E-Business System and Education Technology

\section{Introduction}

There are lots of known web-based instruction platform (WebCT, Blackboard, Claroline, Moodle, etc.), which allow teacher to upload almost any kind of printable document as digital resource into courses. And to read the document, student needs to download the document from the platform and have the software with which that document is created. So it is not very convenient and leads to other problems, for example: (1) incompatibility (reading the document is limit to operating systems or application software), (2) Copyright infringement and insecure (Others can download, copy or update the document), (3) non-real time (reading only wait for the document to download completely), and (4) unmanageable (document is relieved of platform management after it was downloaded).

According to the benefits of flash file (file with SWF extension) [1], such as greater accessibility, compatibility, Internet distribution, web page embeddable, secure, etc. and flash player that is available across a number of operating systems, this paper designs and programs a conversion system, which automatically convert a printable document to a flash file when uploading the printable document on web-based instruction platform.

Corresponding author:

E-mail address: alu@ hainnu.edu.cn; bxie@ hainnu.edu.cn; cjwj@hainnu.edu.cn 


\section{The Technology Scheme}

Reference [2] introduces how to use IPaper API, which is a PHP client library offered by Scribd (http://www.scribd.com/) to upload documents to Scribd server and convert them to flash movie, and then embed them in web applications using Scribd's excellent document viewer. This way is web services, so it is simple, and its defect is that normal work must depend on the unobstructed network connection with Scribd server.

Reference [3] provides some thought for how to use the Documents.Open and Document.ExportAsFixedFormat method to programmatically save an office document as a PDF document. And [4] provides the JAVA source code which uses the open source tool SwfTools to automated converting PDF to SWF. Those solutions can achieve the goal that displaying in Flash based applications, but they are non webbased application.

Flashpaper [5] is a desktop application software developed by Adobe Systems, which convert any printable document (e.g. Microsoft Word, or Excel, or PowerPoint document) to a flash file by printed the document to the virtual printer named Macromedia FlashPaper, and the flash file still retains the formatting, graphics, fonts, special characters, and colors of source documents, regardless of the application and platform used to create the document. Besides graphical user interface, FlashPaper offers Command-line utility flashprinter.exe to trigger conversion process, so it is possible to convert documents to flash programmatically without user assistance. Reference [6] brings up a solution that flashprinter.exe is directly accessible from asp.net. There are some questions: (1) To start the website need to perform additional security configuration which add NETWORK SERVICE and ASP.NET users to administrators groups in order to execute flashpirnter.exe, so the user account under which web page server scripts run has too high rights would lead to insecurity. (2) The absence of thirdparty application process supervision. When flashpaper.exe converts any document, it locates the third-party application which can print documents of the given type and instruct corresponding third-party applications to print the document on the FlashPaper printer. That is to say conversion is performed with the help of external applications. Under the condition that the application prints a document too long or requires user interaction or hangs up during printing, it may prevent the next document from converting; even cause the computer to crash if without closing the application process in time. However, asp.net lacks the strength to cope with application process.

Based above discussion, this paper brings up a solution which combines website module (web form) with desktop application module (win form) to solve those questions. As shown in the fig.1, website module based on IIS7.0 (Internet Information Server) mainly includes two web forms. One is upload.aspx which is responsible for uploading document to file folder on server and passing conversion request to desktop application module, the other is listview.aspx which is responsible for showing the flash files which are converted from Uploaded files for reading. Desktop application module named convert.exe is a win form, which is running in windows services supported by flashpaper and third-party software, and is responsible for getting the specified document from the file folder on server and converting the document to flash file according to the request of upload.aspx. Between two modules, website module is client, desktop application module is server, and both communication by Winsock [7]. This solution have some advantages: (1) the user account under which web form run only has very low rights, because web form have no use for any external application's support; (2) Convert.exe is desk-top application written by $\mathrm{C \#}$, so it is capable of copping with application process; (3) Convert.exe need have the right of administrator, however it only listens and response the conversion request from Upload.aspx, so it is security. (4) The process of conversion runs in background, and weather conversion is successful reflects in listview.aspx, so when the document is uploaded, the uploader immediately obtains feedback and need not wait until converting over. In other words, the user experience is better. 


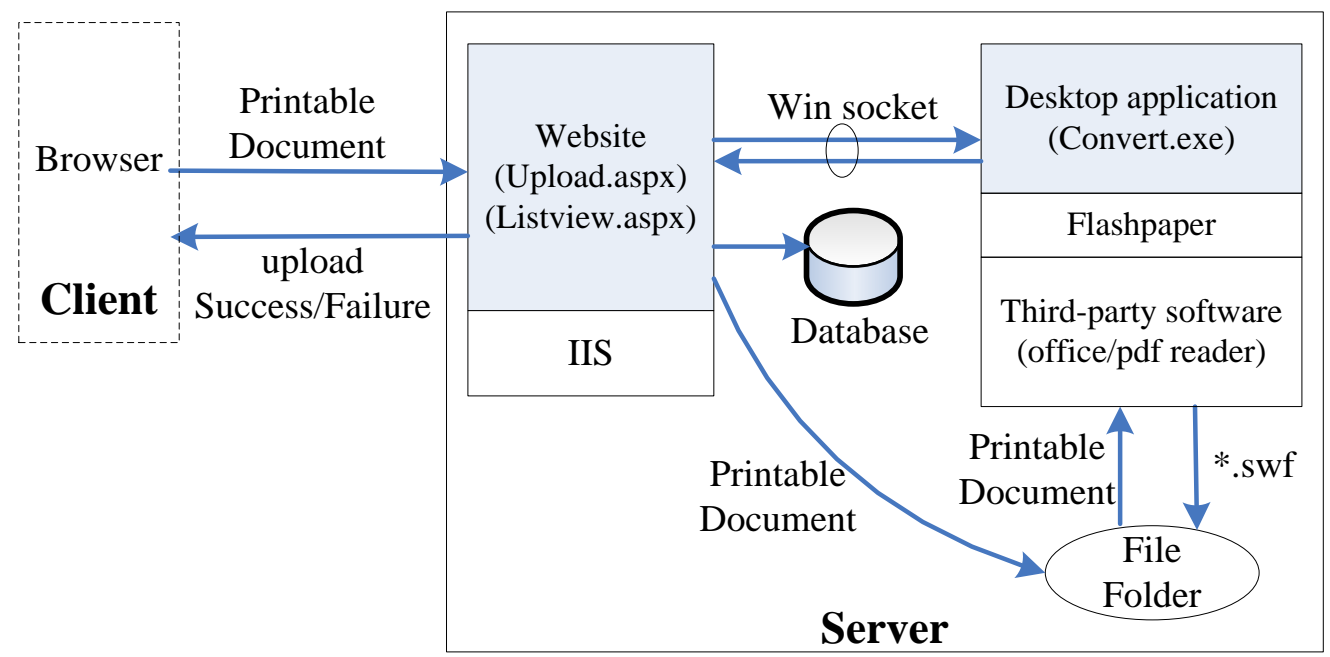

Fig. 1. The Componet of Solution

\section{The Key Implementation}

According to the aforesaid solution, this paper programs and realizes a system based on Microsoft .NET Framework 4.0 and command-line utility flashprinter.exe. And due to space limitations, here only present the algorithm of key procedures and parts of the run-time interface.

\subsection{The implement of upload.aspx}

As shown in the Fig.2, upload.aspx at least includes two controls, one is FileUpload control which is used to locate the local printable document, the other is button control named convert. The mouse-down events of convert button include the following contents: (1) uploads the local printable document which is specified by the textbox of FileUpload control to a specified virtual directory on the website. (2) If the document uploads failed, immediately feedbacks the error message to browser. (3) If the document Uploads succeed, uses the methods of System.Net.Sockets namespace [8] establish a TCP connection with desktop application module (convert.exe), and then sends the document name to convert.exe. (4) If the sending failed, immediately feedbacks the error message to browser. (5) If the sending succeed, inserts a database record consist of ID, Source File Name, File Type, Destination File Name, URL, Start Time of Conversion, Max Conversion Threshold, etc. to the database table named Doc_table, which is built on Microsoft SQL2005. At the moment, Start Time of Conversion is null and Max Conversion Threshold is automatically assigned in accordance with some rule. 


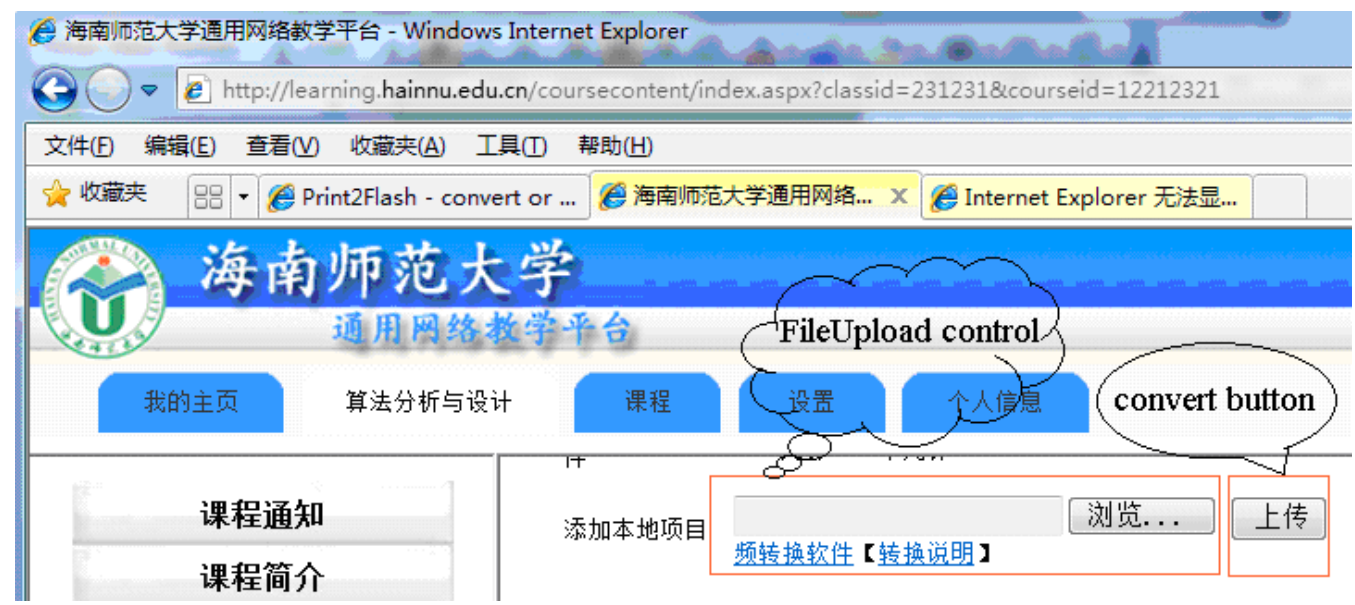

Fig. 2. The user interface of upload.aspx

\subsection{The implement of convert.exe}

Convert.exe uses the methods of System.Net.Sockets namespace to create a socket and listen on a certain IP and Port to waiting for the connection from upload.aspx. When a client connection is accepted, the original socket continuously listens for more incoming connections and a new socket is created to connect to the client socket. If accept succeeds, it forks a new thread to cope with conversion on that new socket. In the new thread, it realizes the following functions:

Step1: Call revc() method to receive the file name of conversion document from upload.aspx.

Step2: According to the file type of conversion document, takes a looks at active process on current system's task manager to see if there is any corresponding third-party application process?

Step3: If the active application process does not exist, selects the corresponding database record on the Doc_table, and then assigns the current time to the Start Time of Conversion of that record, next create a thread which calls Command-line utility flashprint.exe to convert the printable document to a given flash file which agrees with the corresponding Destination File Name.

Step4: If the active application process exists, extracts the start time of active process and then compare the Max Conversion Threshold with the value which is equal to subtract the current system time from that start time.

Step5: If the Max Conversion Threshold is smaller, it call kill() method to kill the active application process, and go to step3.

Step6: If the Max Conversion Threshold is greater or equal, it call sleep() method to wait for the interval time between them, and then go to step2.

The main code of new thread as shown in Fig. 3 


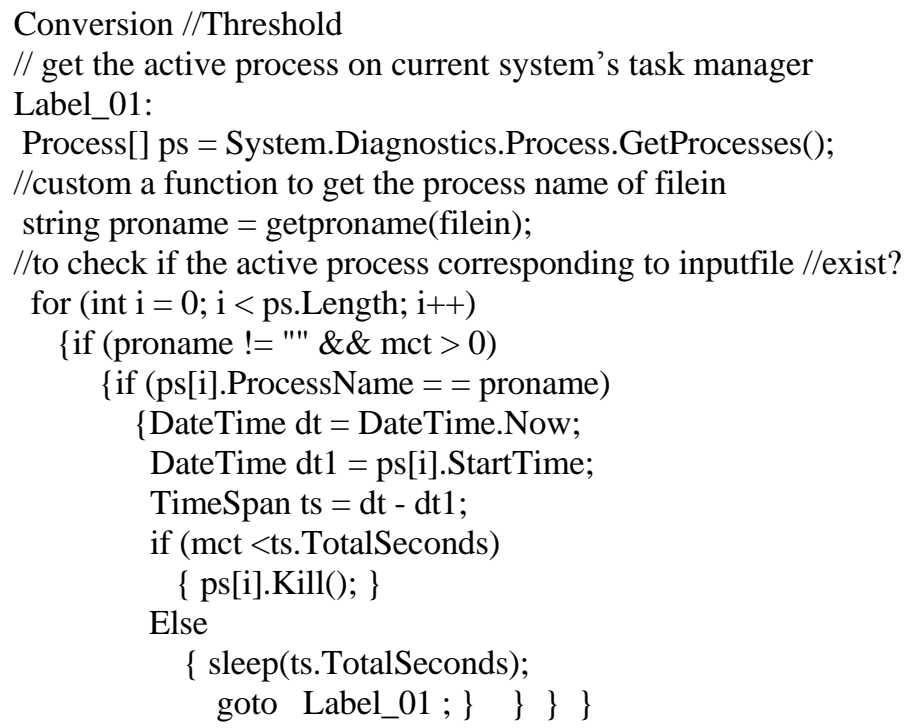

//call command-line utility flashprinter.exe to convert the //document

StringBuilder command = new StringBuilder();

command.Append(ConfigurationManager.AppSettings["proc"].ToString()+"||FlashPrinter.exe ");

command.Append(filein + " ");

command.Append(" -o ");

command.Append(fileout);

Process $\mathrm{p}=$ new Process () ;

p.StartInfo.FileName ="C:IIWINDOWSIIsystem32llcmd.exe";

p.StartInfo.UseShellExecute = false;

p.StartInfo.RedirectStandardInput $=$ true;

p.StartInfo. RedirectStandardOutput = true;

p.StartInfo. RedirectStandardError $=$ true;

p.StartInfo.CreateNoWindow $=$ false;

p.Start();

string strOutput $=$ null;

p.StandardInput.WriteLine(command.ToString());

p.StandardInput.WriteLine("exit");

strOutput $=$ p.StandardOutput.ReadToEnd () ;

Console.WriteLine(strOutput);

p.WaitForExit();

p.Close();

Fig. 3. The source code of new thread 


\section{3. the implement of listview.aspx}

As shown in Fig.4, listview.aspx mainly lists the flash files on webpage for reading in accordance with the records of Doc_table. For every flash file on the webpage, it is a hyperlink and the on-click event of that hyperlink includes the following contents:

Step1: calls exist() method to check if the corresponding flash file exists in the location specified ?

Step2: If the flash file exists, shows the file embedded in a web page as shown in Fig.5.

Step3: If the file does not exist, there are three situations:

- If the Start Time of Conversion of the corresponding record on the Doc_table is null, feedbacks the alarm message that document is converting (as shown in Fig.6).

- If the Start Time of Conversion of the corresponding record on the Doc_table is not null and the Max Conversion Threshold of the corresponding record is greater than the value which subtract the current system time from the Start Time of Conversion of that record, also feedbacks the alarm message that document is converting (as shown in Fig.6).

- Otherwise, feedbacks the error message that conversion is failure. In this case, the uploader needs to delete the hyperlink of that document and upload the document again.

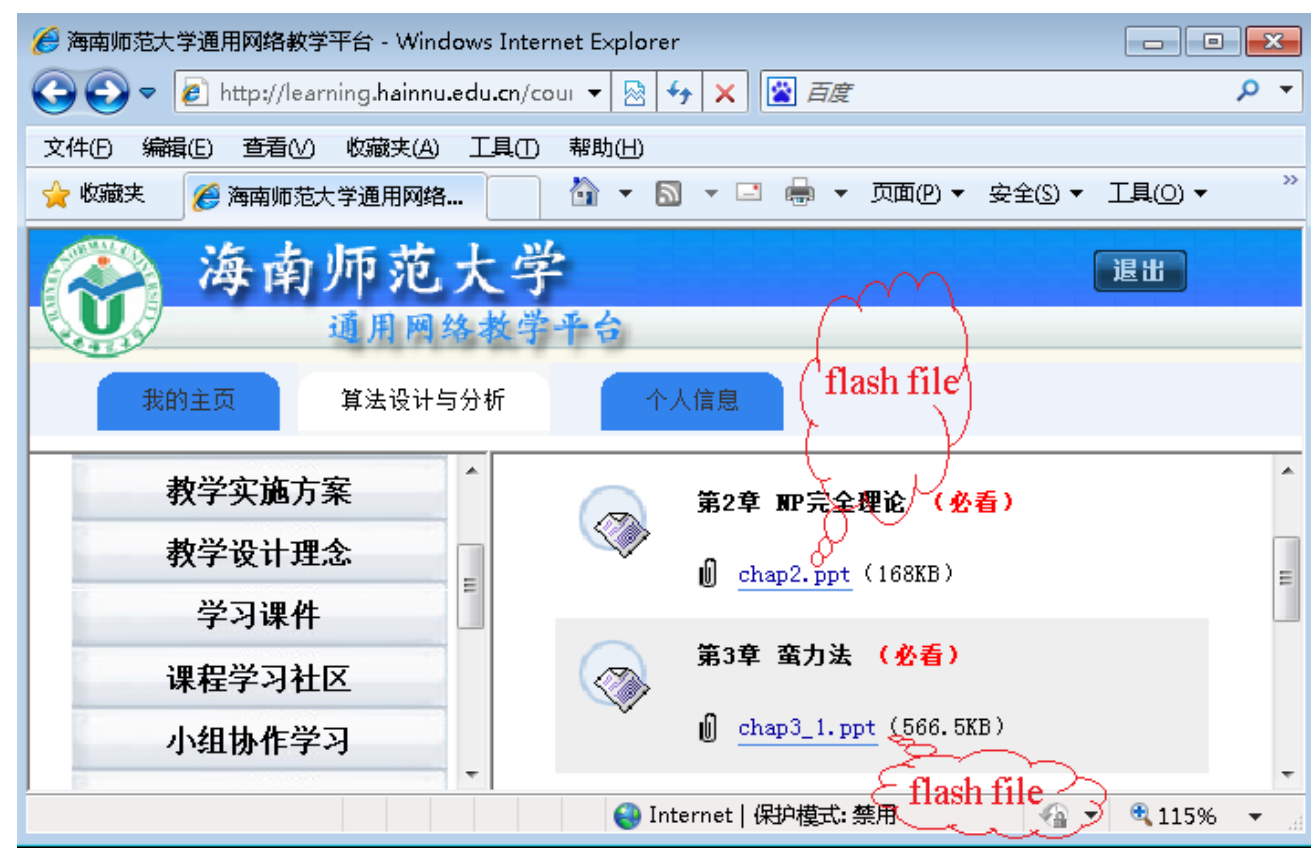

Fig. 4. The list of uploaded documents (flash file) 


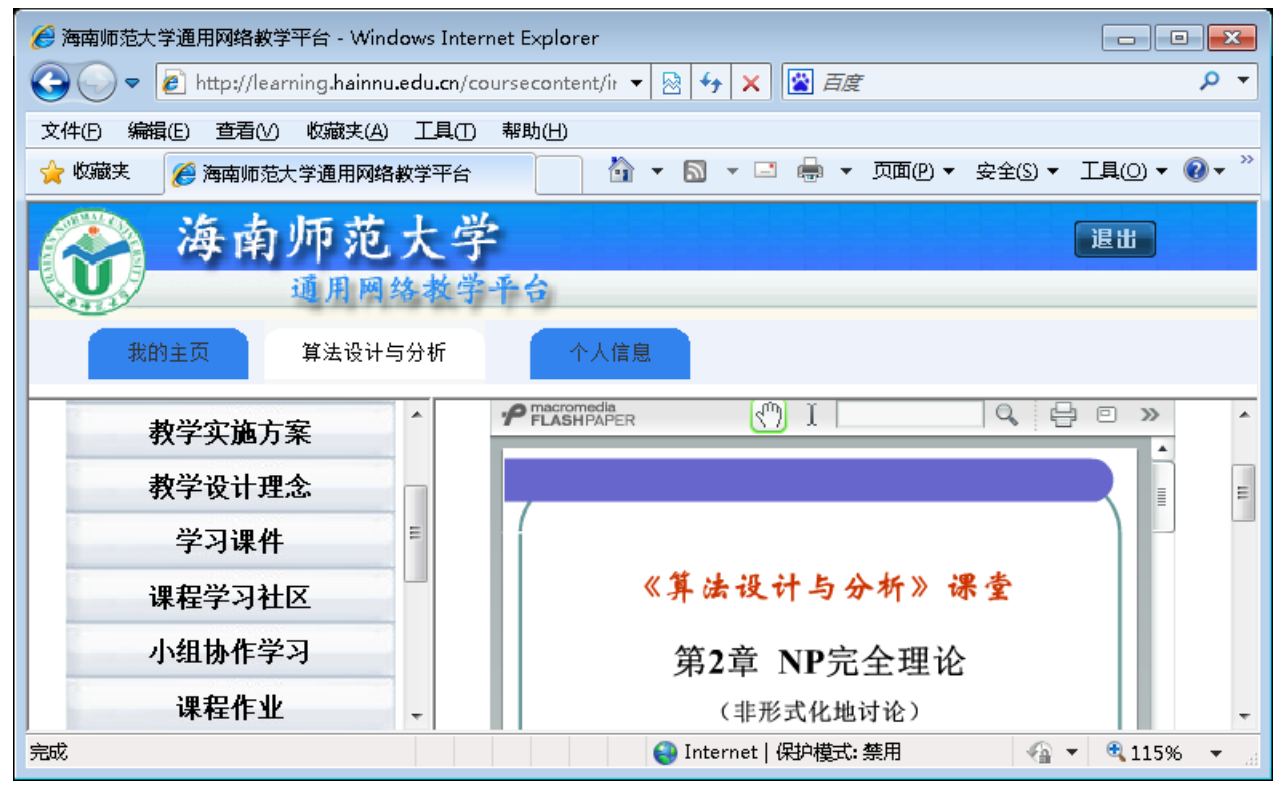

Fig. 5. The document is embed in a web page for reading

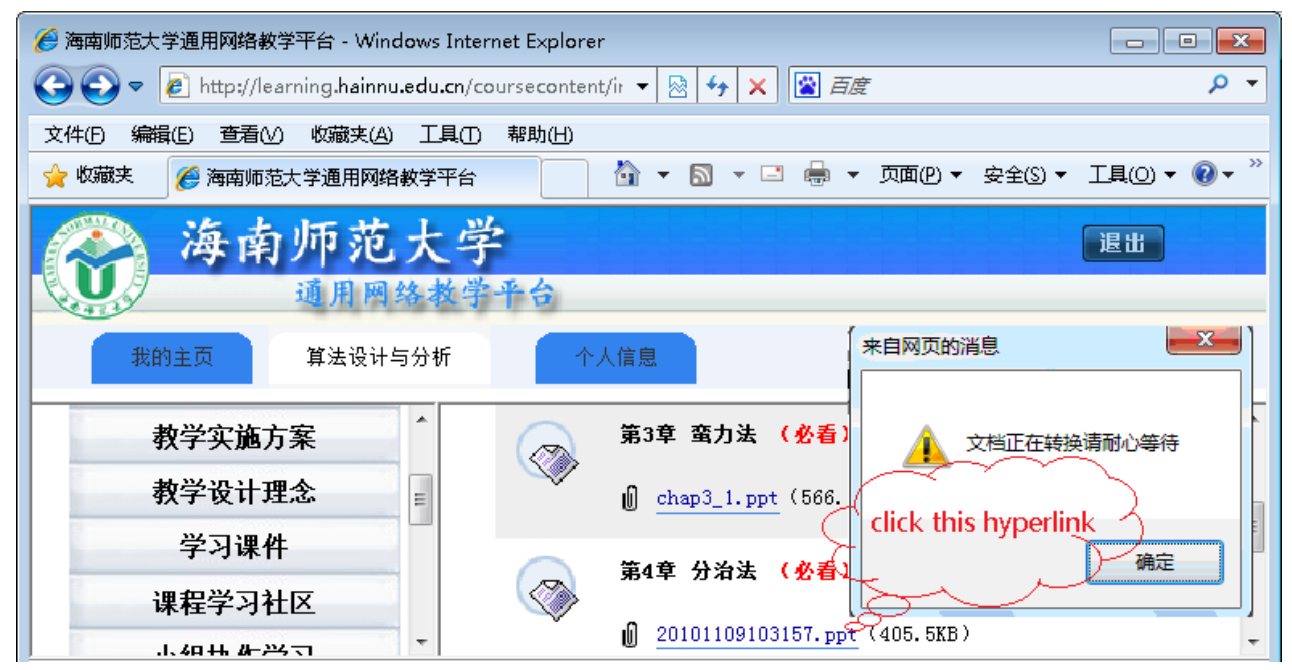

Fig. 6. The alarm message of converting

\section{Conclusion}

After the system which publishes printable documents as flash files on Web-based Network Instruction Platform is deployed, there are a number of benefits as follows: 
- Due to no need the software that created the original document, document can be optionally prevent from downloading, then be limit to embed in a web page and shown inside it altogether with other web page content, so the click rate of a document hyperlink in listview.aspx is approximately equal to the document's reading frequencies. At that point, the reading frequencies can be introduced into the instruction platform to be an important indicator of evaluation of instruction, which can make the instruction platform measurable.

- Because of flash file is a Vector Graphics, its storage space is smaller than original document. On the other hand, it allows online viewers to start watching the presentation without waiting for the entire presentation to download on local machine. So the documents published on the instruction platform can be fast and easy access from anywhere across the internet. That is to say that the platform's user experience is better.

- The documents which have been published on the platform cannot be downloaded, edited, copied, or printing by the viewers. So for the owner of document, the instruction platform is more secure and can protect the author's copyright.

\section{Acknowledgements}

The research project has been supported in part by the Philosophy and Social Sciences Foundation of Hainan Province, China under Grant HNSK06-39, by the Education Department Foundation of Hainan Province, China under project—Develop Web-based Instruction Platform Base on .Net Framework 4.0, and by Youth Research Fund of Hainan Normal University, China under Grant QN0805. I'd like to show my greatest appreciation to those who have given me great support when I was making efforts to complete this paper. My sincere gratitude also goes to reviewers who have provided valuable feedback on this paper.

\section{References}

[1] Print2Flash, http://print2flash.com/help/GettingStarted.php.

[2] H. Hayder, "Using iPaper API to convert office documents to flash movie on the fly", http://hasin.wordpress.com/2009/10/07/ipaper-api-to-convert-office-document-to-flash-movie/,october 2009.

[3] J. Krist, "Saving Word 2007 Documents to PDF and XPS Formats", http://msdn.microsoft.com/enus/library/bb412305(office.12).aspx, April 2007.

[4] L.M. Fang, "Using FlexPaper to display PDF online"(in Chinese), http://blog.csdn.net/flm_0722/archive/2010/07/09/5723505.aspx, July 2010.

[5] FlashPaper, http://www.adobe.com/products/flashpaper/.

[6] W. Zhou, "Calls FlashPaper in Asp.net", http://blog.csdn.net/zhouwen/archive/2010/09/02/5859861.aspx, september 2010.

[7] X.D. Mei, Y.Q Yan, "Network programming technology and practice of Visual C\#" (in Chinese), Beijing:Tsinghua University Press,pp,2008.

[8] Microsoft Corp.,".NET Framework Class Library”, http://msdn.microsoft.com/library/default, 2010. 